MEDICO RESEARCH CHRONICLES

ISSN NO. 2394-3971

DOI No. 10.26838/MEDRECH.2020.7.6.461

Contents available at www.medrech.com

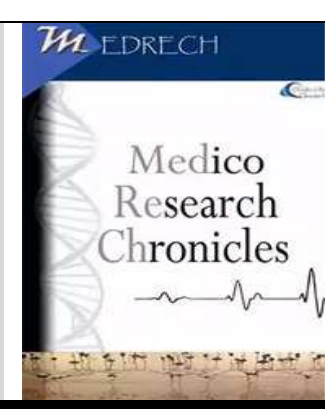

\title{
CARDIO-DIABETIC PROTECTIVE EFFECT OF TELMISARTAN
}

\author{
Shiladitya Nandi, Sourav Nag, Trinanjan Ash, V Chandra Shekhar \\ 1. 6A, Ballygunge Place, Kolkata-700019 \\ 2. Purbachal, Barasat,Near Narayan Hridhlay,Kolkata-700124 \\ 3. 60/1 Swami Sibananda Road, Choudhury Para, P.O-Barasat, Ps-Barasat, Kolkata-700124 \\ 4. Satyanarayana Clinic. Beside Ekashila Park. Balasamudram. Hanamkonda
}

\section{ARTICLE INFO}

\section{Article History}

Received: November 2020

Accepted: December 2020

Keywords: Telmisartan, antihypertensive, Diabetes, PPAR $\gamma$ receptor

Corresponding author* Shiladitya Nandi *
The antihypertensive drugs that are being used currently were primarily designed and developed to affect cellular and biochemical mechanisms that lead to increased blood pressure and do not address the underlying contributory factors like, disordered carbohydrate and lipid metabolism that often accompanies hypertension as part of the metabolic syndrome. The metabolic syndrome eventually leads to co-morbid conditions i.e., hypertension and type 2 diabetes mellitus. Both these conditions mechanistically share the common pathophysiological pathways in people who suffer from metabolic syndrome. PPAR- $\gamma$ plays an important role in regulating carbohydrate and lipid metabolism and ligands for PPAR $\gamma$ can improve insulin sensitivity, reduce triglyceride levels, and decrease the risk for atherosclerosis. Telmisartan is a wellestablished angiotensin II type 1 receptor (AT1) blocker along with an activator of the PPAR $\gamma$ receptor. Telmisartan has several pharmacological properties that distinguish it from other ARBs, like, the structural resemblance with pioglitazone which is a PPAR $\gamma$ ligand that is approved for the treatment of type 2 diabetes. Thus, telmisartan seems to be a suitable candidate for the management of $\mathrm{CV}$ risk reduction in patients with atherothrombotic disease or diabetes mellitus with endorgan damage. This article will discuss the mechanistic aspect of telmisartan that supports its utility in cardiac protection along with the outcome of different clinical trials.

O2020, www.medrech.com

All currently available classes of antihypertensive drugs were developed before it was widely recognized that increased blood pressure is closely associated with insulin resistance and dyslipidemia and well before public health authorities established diagnostic criteria for the metabolic syndrome. Thus, the antihypertensive drugs in use today were designed primarily to affect cellular and biochemical mechanisms contributing to 
increased blood pressure and not to address the disordered carbohydrate and lipid metabolism that often accompanies hypertension as part of the metabolic syndrome. Given the major impact of metabolic syndrome on cardiovascular disease morbidity and mortality, the availability of antihypertensive agents that also improve insulin resistance and dyslipidemia are of considerable clinical value $^{1}$. Hypertension and type 2 diabetes mellitus seem to be two aspects of common pathophysiological pathways, especially in people who suffer from metabolic syndrome. Diabetes mellitus is one of the major risk factors for atherosclerosis, an inflammatory disease of the arterial wall, in which leukocytes and oxidized lipoproteins accumulate leading to the formation of fatty streaks and atherosclerotic plaques. A key feature common to all end-stage forms of diabetes is hyperglycemia, which may in part account for macrovascular complications. Both insulin resistance and subsequent hyperinsulinemia are key features of type 2 diabetes. Type 2 diabetes is often preceded by or associated with metabolic syndrome, a risk factor complex for cardiovascular disease consisting of atherogenic dyslipidemia, insulin resistance accompanied by glucose intolerance, abdominal obesity, a proinflammatory and prothrombotic state, and hypertension. Type 2 diabetes is associated with hyperlipidemia (elevated triglycerides, decreased high-density lipoprotein (HDL), and increased low-density lipoprotein (LDL) levels. Obesity and increased visceral adiposity present as the most important pathogenetic factors as they lead to chronic low-grade inflammation which along with oxidative stress in adipose tissue finally causes increased production of angiotensinogen and angiotensin II. Furthermore, in addition to the oxidation of lipoproteins hyperglycemia may lead to glycation leading to the formation of pro-atherogenic glycoxidation products. It is proposed that impaired mitochondrial activity results in dysregulation of intracellular fatty acid metabolism. Increased intracellular fatty acids may activate $\mathrm{PKCb}$ and $\mathrm{d}$ via DAG and thereby lead to serine phosphorylation of the insulin receptor substrate (IRS)-1, resulting in reduced glucose transport. These defects seem to be closely related to increased body fat. Indeed, visceral abdominal fat has important endocrine functions, leading to increased serum levels of TNF- $\alpha$, interleukin- 6 , plasminogen activator inhibitor (PAI-1), and reduced expression of adiponectin. In humans, insulin resistance has been associated with low expression of IRS-1. Also, patients displayed reduced serum levels of adiponectin and reduced gene expression of peroxisome proliferator-activated receptor- $\gamma$ (PPAR $\gamma$ ), fatty acid-binding protein (FABP) aP2, and lipoprotein lipase (LPL) in adipose tissue ${ }^{2}$. As the primary site for energy storage, adipose tissue plays an important role in energy balance and metabolism ${ }^{3}$. PPAR- $\gamma$ plays an important role in regulating carbohydrate and lipid metabolism and that ligands for PPAR $\gamma$ can improve insulin sensitivity, reduce triglyceride levels, and decrease the risk for atherosclerosis $^{1}$. Adiponectin serum levels have been demonstrated to be inversely correlated with insulin resistance and inflammatory state. Adipokines are cytokines produced by adipose tissue; they may be related to the metabolic syndrome and have pro- as well as antiatherogenic effects. Interestingly, PPAR $\gamma$ agonists are known to promote adipocyte differentiation and also to increase adiponectin transcription and serum levels. However, clinical data suggest that PPAR $\gamma$ agonists may rather promote cardiovascular adverse events than be atheroprotective. However, telmisartan -an angiotensin receptor blocker administered in hypertensive patients - has been demonstrated to increase adiponectin transcription in human adipocytes $^{2}$.

The interesting structural resemblance between telmisartan, an angiotensin II (Ang II) 
type 1 receptor antagonist approved for the treatment of hypertension, and pioglitazone, a PPAR $\gamma$ ligand approved for the treatment of type 2 diabetes supported the possibility that certain molecules might have the capacity not only to block the Ang II receptor, a key cell surface receptor involved in the regulation of blood pressure but also to activate PPAR $\gamma$, an intracellular nuclear hormone receptor involved in the regulation of carbohydrate and lipid metabolism. Theoretically, such bifunctional molecules could treat both the hemodynamic and biochemical features of the metabolic syndrome and have greater potential for preventing atherosclerotic cardiovascular disease than conventional antihypertensive agents. Moreover, given that blockade of the renin-angiotensin system can inhibit renal sodium reabsorption and attenuate the fluid retention and edema associated with peripheral vasodilators, such molecules could also lead to the development of new antidiabetic PPAR $\gamma$ ligands with improved safety profiles ${ }^{1}$. Telmisartan is a well-established angiotensin II type 1 receptor (AT1) blocker along with an activator of the PPAR $\gamma$ receptor.

Telmisartan is one of the widely used antihypertensive agents, which has demonstrated favorable safety and tolerability profiles, both alone and in combination therapies. It has several pharmacological properties that distinguish it from other ARBs - the longest plasma half-life, highest lipophilicity, and strongest receptor binding affinity in class. Telmisartan is, therefore has a broad indication for $\mathrm{CV}$ risk reduction in patients with atherothrombotic disease or diabetes mellitus with end-organ damage. Telmisartan can effectively improve insulin sensitivity in patients with hypertension and because of its additive PPAR $\gamma$ agonistic action, it can improve vascular inflammation, reduce visceral fat, and increase serum adiponectin along with reducing the elevated $\mathrm{BP}$ in essential hypertensive patients ${ }^{4}$. Metaanalyses of hypertension studies show that blockade of the angiotensin receptor blockers (ARBs) is more effective than other classes viz., rennin angiotensin system by ACE inhibitors, of blood pressure-lowering medications for the prevention of incident diabetes ${ }^{5}$.

The Telmisartan Randomised Assessment Study in ACE iNtolerant subjects with cardiovascular Disease (TRANSCEND) study assessed whether the angiotensinreceptor blocker telmisartan would be effective in 5926 patients that were intolerant to Angiotensin-converting enzyme (ACE) inhibitors with cardiovascular disease or diabetes with end-organ damage. It was found that Mean blood pressure was lower in the telmisartan group than in the placebo group throughout the study (weighted mean difference between groups 4.0/2.2 [SD 19.6/12.0] $\mathrm{mm} \mathrm{Hg}) .465(15 \cdot 7 \%)$ patients experienced the primary outcome in the telmisartan group compared with 504 (17.0\%) in the placebo group (hazard ratio $0.92,95 \%$ CI $0 \cdot 81-1 \cdot 05, p=0 \cdot 216)$. One of the secondary outcomes-a composite of cardiovascular death, myocardial infarction, or strokeoccurred in $384(13.0 \%)$ patients on telmisartan compared with $440(14.8 \%)$ on placebo $\quad(0.87, \quad 0.76-1.00, \quad \mathrm{p}=0.048$ unadjusted; $\mathrm{p}=0.068$ after adjustment for multiplicity of comparisons and overlap with the primary outcome). 894 (30.3\%) patients receiving telmisartan were hospitalized for a cardiovascular reason, compared with 980 $(33.0 \%)$ on placebo (relative risk $0.92,95 \%$ CI 0.85-0.99; $\mathrm{p}=0.025)$. Fewer patients permanently discontinued study medication in the telmisartan group than in the placebo group $(639 \quad[21.6 \%]$ vs 705 [23.8\%]; $\mathrm{p}=0.055)$; the most common reason for permanent discontinuation was hypotensive symptoms (29 [0.98\%] in the telmisartan group vs 16 [0.54\%] in the placebo group). It was concluded that Telmisartan was well tolerated in patients unable to tolerate ACE inhibitors. Although the drug had no 
significant effect on the primary outcome of this study, which included hospitalizations for heart failure, it modestly reduced the risk of the composite outcome of cardiovascular death, myocardial infarction, or stroke ${ }^{6}$.

Kubik et al. carried out a study to estimate changes in insulin resistance and plasma concentrations of adipokines after long-term antihypertensive treatment with telmisartan in obese hypertensive patients. 34 previously untreated obese adults with arterial hypertension were enrolled. Glucose cellular uptake ( $\mathrm{M}$ value) and the $\mathrm{M}$ to insulin ratio (M/I value) were measured by euglycemichyperinsulinemic clamp technique, body fat content (by dual-energy X-ray absorptiometry method), as well as plasma concentrations of selected adipokines and cytokines, were estimated before and after 6-month telmisartan therapy in 25 patients who completed the study. Telmisartan therapy was followed by a $14.2 \%$ decrease of systolic and by $19.6 \%$ decrease in diastolic blood pressure. Body fat mass did not change significantly. Both $\mathrm{M}$ and $\mathrm{M} / \mathrm{I}$ values (by 24.4 and by $38.6 \%$, respectively), as well as plasma levels of total and high-molecular-weight adiponectin (by 10.8 and by $23.5 \%$, respectively), increased significantly. Plasma concentrations of highsensitivity $\mathrm{C}$ reactive protein and interleukin- 8 decreased significantly, while those of interleukin- 6 and tumor necrosis factor- $\alpha$ tended to decline. Telmisartan monotherapy improves cardiometabolic profile in obese hypertensive patients by improving insulin sensitivity and increasing plasma adiponectin concentration, including its high-molecularweight fraction, and by suppressing microinflammation ${ }^{7}$. Takagi et al., performed the meta-analysis of randomized controlled trials to determine whether telmisartan improves metabolic parameters in metabolic syndrome covering 10 reports of RCTs, that have enrolled a total of 546 patients with metabolic syndrome. Pooled analysis suggested significant reductions in \% changes of fasting glucose (standardized mean difference, (-)0.51; 95\% confidence interval [CI], (-)0.96 to (-)0.06; P 1/4 .03), insulin (0.23 ; $95 \%$ CI, (-) 0.40 to $(-) 0.06 ;$ P $1 / 4.008)$, glycosylated hemoglobin $(-0.26 ; 95 \% \mathrm{CI}$, (0.44 to (-)0.08; P 1/4 .005), and homeostasis model assessment index $(-0.22 ; 95 \%$ CI (0.39 to $(-) 0.05 ;$ P $1 / 4.01)$; and a significant increase in $\%$ changes of adiponectin $(0.75$; $95 \%$ CI, 0.40 to $1.09 ; \mathrm{P}<.0001)$ among patients with metabolic syndrome randomized to telmisartan versus control therapy. Telmisartan therapy significantly improves the metabolic parameters in patients with metabolic syndrome ${ }^{8}$.

Sasaki et al. compared the effects of telmisartan and olmesartan in 20 patients with chronic heart failure and metabolic syndrome. It was found that there were no fluctuations in either blood pressure in the late evening or the outpatient room; nor were there fluctuations in heart rate in both the groups. Simultaneously, neither serum high-density lipoprotein cholesterol nor fasting blood sugar levels differed significantly between groups. However, telmisartan had more beneficial effects on glucose and lipid profiles in patients with relatively high $\mathrm{HbA} 1 \mathrm{c}$, serum total and low-density lipoprotein cholesterol, and triglyceride levels. Thus, it was concluded that telmisartan was more beneficial than olmesartan for controlling blood pressure in the early morning, as well as for improving glucose and lipid profiles in patients with hypertension, chronic heart failure, and metabolic syndrome ${ }^{9}$.

Chang et al investigated the effectiveness of telmisartan in improving left ventricular remodeling in diabetic humans and rats. In this longitudinal, prospective study, 15 diabetic patients were enrolled and were given telmisartan (20 mg/day) for 12 weeks. It was found that Telmisartan improved the hyperglycemia-induced cardiac fibrosis through the PPAR $\delta /$ STAT3 pathway ${ }^{10}$.

\section{CONCLUSION}


The incidence of diabetes and diabetesrelated atherosclerosis has dramatically increased over the past years. Many of the proatherogenic mechanisms of diabetes are related to hyperglycemia and thereby promote macrovascular complications. Considering the overlap of pathogenetic mechanisms of diabetes and atherosclerosis, it seems promising to identify therapeutic targets shared by both diseases. Activation of PPAR $\gamma$ by telmisartan makes it an ARBs that ameliorate insulin resistance and dyslipidemia as well as hypertension and that could potentially provide even more effective options for preventing target-organ damage and cardiovascular disease in patients with hypertension, diabetes, or both. Thus, telmisartan is a drug having dual benefits in the management of cardiac as well as diabetic patients.

\section{REFERENCES}

1. Benson SC, Pershadsingh HA, Ho CI, et al. Identification of Telmisartan as a Unique Angiotensin II Receptor Antagonist with Selective PPAR $\gamma$-Modulating Activity. Hypertension. 2004;43(5):993-1002. doi:10.1161/01.HYP.0000123072.34629.57

2. Gleissner CA, Galkina E, Nadler JL, Ley K. Mechanisms by which diabetes increases cardiovascular disease. Drug Discov Today Dis Mech. 2007;4(3):131-140. doi:10.1016/j.ddmec.2007.12.005

3. Shiota A, Shimabukuro M, Fukuda D, et al. Activation of AMPK-Sirt1 pathway by telmisartan in white adipose tissue: A possible link to anti-metabolic effects. Eur $J \quad$ Pharmacol. 2012;692(1-3):84-90. doi:10.1016/j.ejphar.2012.07.026

4. Arumugam S, Sreedhar R, Thandavarayan RA, et al. Angiotensin receptor blockers: Focus on the cardiac and renal injury. Trends Cardiovasc Med. 2016;26(3):221-
228. doi:10.1016/j.tcm.2015.06.004

5. Barzilay JI, Gao P, Rydén L, et al. Effects of telmisartan on glucose levels in people at high risk for cardiovascular disease but free from diabetes: The TRANSCEND study. Diabetes Care. 2011;34(9):1902-1907. doi:10.2337/dc11-0545

6. Inoue T, Morooka T, Moroe K, Ikeda H, Node K. Effect of telmisartan on cholesterol levels in patients with hypertension - Saga Telmisartan Aggressive Research (STAR). Horm Metab Res. 2007;39(5):372-376. doi:10.1055/s2007-976544

7. Kubik M, Chudek J, Adamczak M, Wiecek A. Telmisartan improves cardiometabolic profile in obese patients with arterial hypertension. Kidney Blood Press Res. 2012;35(4):281-289. doi: 10.1159 /000334951

8. Takagi H, Niwa M, Mizuno Y, Goto SN, Umemoto T. Telmisartan as a metabolic sartan: The first meta-analysis of randomized controlled trials in metabolic syndrome. $J$ Am Soc Hypertens. 2013;7(3):229-235. doi:10.1016/j.jash.2013.02.006

9. Sasaki T, Noda Y, Yasuoka Y, et al. Comparison of the effects of telmisartan and olmesartan on home blood pressure, glucose, and lipid profiles in patients with hypertension, chronic heart failure, and metabolic syndrome. Hypertens Res. 2008;31(5):921-929.

doi:10.1291/hypres.31.921

10. Chang WT, Cheng JT, Chen ZC. Telmisartan improves cardiac fibrosis in diabetes through peroxisome proliferatoractivated receptor $\delta$ (PPAR $\delta$ ): From bedside to bench. Cardiovasc Diabetol. 2016;15(1):1-9. doi:10.1186/s12933-0160430-5 